TRANSACTIONS OF THE

AMERICAN MATHEMATICAL SOCIETY

Volume 356, Number 12, Pages 4951-4968

S 0002-9947(04)03475-0

Article electronically published on April 16, 2004

\title{
SPECTRAL PROPERTIES AND DYNAMICS OF QUANTIZED HENON MAPS
}

\author{
BRENDAN WEICKERT
}

\begin{abstract}
We study a generalization of the Airy function, and use its properties to investigate the dynamics and spectral properties of the unitary operators on $L^{2}(\mathbf{R})$ of the form $U_{c}:=F e^{i(q(x)+c x)}$, where $q$ is a real polynomial of odd degree, $c$ is a real number, and $F$ is the Fourier transform. We show that $U_{c}$ is a quantization of the classical Henon map

$$
\begin{aligned}
f_{\lambda}: \mathbf{R}^{2} & \rightarrow \mathbf{R}^{2}, \\
(x, y) & \mapsto\left(y+q^{\prime}(x)+c,-x\right),
\end{aligned}
$$

and show that for $c>0$ sufficiently large, $U_{c}$ has purely continuous spectrum. This fact has implications for the dynamics of $U_{c}$, which are shown to correspond when the condition is satisfied to the dynamics of its classical counterpart on $\mathbf{R}^{2}$.
\end{abstract}

\section{INTRODUCTION}

In studying complex dynamics on infinite-dimensional spaces, it is natural, in order to reduce the complexity of the problem, to place some restrictions on the map being iterated. We might begin by requiring that the map be a linear selfmap of, say, a Banach space or a separable Hilbert space. However, this restriction does not suffice to produce an interesting theory. If we let $M$ be a compact metric space, $f: M \rightarrow M$ a continuous self-map, and $B$ the backward shift on the Banach space $l^{\infty}(M)$, for instance, it is well known that the usual symbolic dynamics trick of identifying a point with its orbit shows that $f$ is topologically conjugate to a restriction of $B$. Thus $B$ exhibits all the known behavior of finite-dimensional dynamical systems on compact spaces. Since any compact metric space embeds in separable Hilbert space $\mathcal{H}$, one may consider $B$ as acting on $l^{\infty}(\mathcal{H})$ to obtain a universal example.

A further restriction is thus called for, and so we confine ourselves to unitary maps on separable Hilbert space $\mathcal{H}$. The fact that this class of maps contains the quantizations of finite-dimensional Hamiltonian systems (such as the real Henon map), combined with the interplay between the spectral properties and dynamics of its members, makes it a natural object of study. Let us focus on the quantizations of Hamiltonian systems on $M^{\prime}=M \times \mathbf{R} \ni(x, y)$, where $M$ is either $\mathbf{T}$ or $\mathbf{R}$. Thus we have a differentiable function $H: M^{\prime} \rightarrow \mathbf{R}$ (in general, $H=H_{t}$ is time-dependent), a Hamiltonian vector field $X=X_{H}:=\left(H_{y},-H_{x}\right)$, and a flow $\phi_{t}: M^{\prime} \rightarrow M^{\prime}$

Received by the editors January 15, 2003 and, in revised form, July 3, 2003.

2000 Mathematics Subject Classification. Primary 32H50; Secondary 37N20.

(C)2004 American Mathematical Society 
satisfying

$$
\begin{aligned}
\frac{\partial}{\partial t} \phi_{t} & =X \circ \phi_{t}, \\
\phi_{0} & =\mathrm{Id} .
\end{aligned}
$$

We then quantize this system by replacing $H$ with a self-adjoint operator on $L^{2}(M)$, which we also denote by $H$, and defining $U_{t}$ as the unitary solution operator to the Schrodinger equation

$$
\begin{aligned}
i \frac{\partial}{\partial t} U_{t} & =H, \\
U_{0} & =\mathrm{Id} .
\end{aligned}
$$

One might ask if the broad features of the long-term dynamics of $\phi_{t}$ have analogues in the dynamics of $U_{t}$. If $\phi_{t}$ has an open, bounded, invariant subset, for instance, does $U_{t}$ also exhibit recurrent behavior? Insofar as the recurrence properties of $U_{t}$ are connected to properties of its spectrum (see [AS]), we would be looking for instance for an eigenvector of $U_{t}$. One may also fix an operator $U:=U_{t_{0}}$ and consider the corresponding discrete dynamics.

If $H$ is time-independent, we have the representation $U_{t}:=e^{-i t H}$, where the meaning of the exponential is given by the spectral theorem's functional calculus. If $H$ is time-dependent, but the family $U_{t}$ is strongly continuous, then Stone's Theorem provides a self-adjoint operator $A$ such that, again, $U_{t}$ has the representation $U_{t}=$ $e^{i t A}$. See for instance [RS]. Given a fixed unitary operator $U$, if $U=V^{-1} e^{i x} V$ is its spectral representation, then trivially $U=e^{i A}$, where $A=V^{-1} x V$. However, we will seldom have available a representation of this form, since typically the unitary operator $V$ is not known. This might be a good point at which to make the following fundamental observation about unitary dynamics on infinite-dimensional Hilbert space: the mere knowledge that a unitary operator is conjugate to a multiplication operator on some space conveys little information about its dynamics. What is required is spectral information about the operator. This fact distinguishes the infinite-dimensional problem from the trivial finite-dimensional problem.

Returning to the problem of quantization, there is in general no canonical way to perform the above-mentioned replacement of $H$ by a (possibly time-dependent) element of $\mathcal{A}\left(L^{2}(M)\right)$, and there is vast literature on the subject (see [KS]). The problem is essentially to construct a nondegenerate Lie algebra homomorphism from a reasonable algebra (under the Poisson bracket) of real-valued functions on $M^{\prime}$ to $\mathcal{A}\left(L^{2}(M)\right)$ (considered as a Lie algebra under the usual Lie bracket of operators). It is a classical result that the nondegeneracy requirement allows us to assume, without loss of generality, that our desired homomorphism would satisfy

$$
\begin{aligned}
& x \mapsto M_{x}=: x \in \mathcal{A}\left(L^{2}(M)\right), \\
& y \mapsto-i d / d x=: y \in \mathcal{A}\left(L^{2}(M)\right) .
\end{aligned}
$$

Here $M_{x}$ denotes multiplication by $x$. This uniquely determines the map, called the Dirac quantization. However, it is quite easy to show the impossibility of constructing such a homomorphism on any reasonably-sized algebra of functionsthat is, the Dirac quantization does not exist. Therefore one weakens the desirata, and seeks instead to construct a map that preserves the algebra structure only asymptotically in some real parameter, usually denoted by $\hbar$. This may easily be done-the Weyl quantization accomplishes it, for instance-but not in a canonical 
way. We refer the reader to the literature for a full discussion of the subtleties involved here. In our cases, however, we will be treating Hamiltonian functions that are simple enough that there is no ambiguity about what their quantizations should be, as they lie in function algebras small enough that the Dirac quantization may be defined, and where the other, weaker notions of quantization coincide. We will also assume in what follows that $\hbar=1$.

A simple way to produce a unitary operator on $L^{2}(M)$ with nontrivial dynamics is to compose two noncommuting operators with known spectral properties. Any operator of the form

$$
e^{i g(x)} e^{i f(y)}
$$

is an example. Operators of the form

$$
e^{-i g(x)} e^{-i y^{2} / 2}
$$

are of particular interest to us, because they describe free motion with "kicks" to the momentum. Let

$$
H=H_{t}= \begin{cases}y^{2} / 2 & 0 \leq t<1, \\ g(x) & 1 \leq t<2,\end{cases}
$$

extended by periodicity. This notation describes both a time-dependent Hamiltonian function on $M^{\prime}$ and the element of $\mathcal{A}\left(L^{2}(M)\right)$ that quantizes it. Solving the Schrodinger equation with this operator $H$ gives a unitary solution $U_{t}$ such that

$$
U:=U_{2}=e^{-i g(x)} e^{-i y^{2} / 2} .
$$

Alternatively, let

$$
H=H_{t}:=y^{2} / 2+g(x) \sum_{n=1}^{\infty} \delta(t-n) .
$$

Solving the Schrodinger equation with this Hamiltonian again produces a family $U_{t}$ such that $U_{1}=U$. This latter Hamiltonian has the form of a traditional Schrodinger Hamiltonian,

$$
H=y^{2} / 2+V_{t}(x),
$$

which describes the motion of a particle in the potential $V_{t}$. In our case, we interpret the Hamiltonian as describing free motion $(V=0)$, interrupted periodically by "kicks", or instantaneous changes in the momentum. A great deal of attention has been paid to operators of this form. The so-called quantized kicked rotor, for instance, is a much-studied element of $\mathcal{U}\left(L^{2}(\mathbf{T})\right)$ whose dynamics and spectral properties are still largely unknown. It is defined by

$$
U_{\alpha}:=e^{-\pi i \alpha y^{2}} e^{-i \cos x},
$$

where $\alpha \in \mathbf{R} \backslash \mathbf{Q}$. See $[\mathrm{Be}]$ for a discussion of this operator. Fornaess $[\mathrm{Fo}$ has studied the operator

$$
U_{\alpha}:=e^{-2 \pi i \alpha y} e^{-i v(x)} \in \mathcal{U}\left(L^{2}(\mathbf{T})\right)
$$

in the case where $\alpha$ is the golden mean $(\sqrt{5}+1) / 2$, where $v(x)$ is a tent map on the circle, and the present author [W1] has studied the same operator for general $\alpha \in \mathbf{R}$. This work is characterized by small denominator problems related to the Diophantine properties of $\alpha$, recalling the similar problems encountered in the study of local holomorphic germs of self-maps of $\mathbf{C}$ near fixed points; see $[\mathbf{C G}$. 
Finally, Fornaess and the author [FW] have studied

$$
U_{c}:=e^{i y^{2} / 2} e^{-i\left(x^{3} / 3-x^{2}+c x\right)} \in \mathcal{U}\left(L^{2}(\mathbf{R})\right)
$$

for $c>0$. It quantizes the classical Henon map on $\mathbf{R}^{2}$. Using the fact that, up to a multiplicative constant of absolute value one, the Fourier transform may be written as

$$
F=e^{-i x^{2} / 2} e^{-i y^{2} / 2} e^{-i x^{2} / 2},
$$

and replacing $U_{c}$ by unitary conjugates and inverses, we take instead

$$
V_{c}=F e^{i\left(x^{3} / 3+c x\right)},
$$

which, as described in [FW], quantizes the classical Henon map

$$
\begin{aligned}
\phi_{c}: \mathbf{R}^{2} & \rightarrow \mathbf{R}^{2}, \\
(x, y) & \mapsto\left(y+x^{2}+c,-x\right) .
\end{aligned}
$$

It is easy to show that for $c$ sufficiently large and positive, the iterates of $\phi_{c}$ map all points of $\mathbf{R}^{2}$ towards $(\infty,-\infty)$. It is natural to ask whether an analogue holds in the quantum system. Thus we have the following theorem [FW]:

Theorem. For $V_{c} \in \mathcal{U}\left(L^{2}(\mathbf{R})\right)$ as above, if $c>0$ is sufficiently large, then for all $\psi \in L^{2}(\mathbf{R}), \epsilon>0$, and $r \in \mathbf{R}$, there exists an $N$ such that for all $n \geq N$, we have

$$
\begin{aligned}
\left\|V_{c}^{n} \psi\right\|_{(-\infty, r]} & <\epsilon, \\
\left\|F V_{c}^{n} \psi\right\|_{[-r, \infty)} & <\epsilon .
\end{aligned}
$$

In particular, $V_{c}^{n} \rightarrow 0$ as $n \rightarrow \infty$ in the weak operator topology, which implies that $V_{c}$ has a purely continuous spectrum.

The proof makes use of specific properties of the Airy function, defined by the integral formula

$$
A i(x)=\frac{1}{2 \pi} \int_{\mathbf{R}} e^{i\left(t^{3}-x t\right)} d t,
$$

interpreted in the principal value sense. In this paper, we wish to generalize this theorem to the case

$$
V_{c}=F e^{i(q(x)+c x)},
$$

where $q$ is a polynomial of odd degree at least three, with positive leading coefficient. If $q$ is real, then, as we shall describe in the next section, $V_{c}$ quantizes the Henon map

$$
\begin{aligned}
\phi_{c}: \mathbf{R}^{2} & \rightarrow \mathbf{R}^{2}, \\
(x, y) & \mapsto\left(y+q^{\prime}(x)+c,-x\right) .
\end{aligned}
$$

Once again, if the leading coefficient of $q$ is positive, then for $c$ sufficiently large it is easy to show that all points are mapped by the iterates of $\phi_{c}$ towards $(\infty,-\infty)$. We wish to prove that the above theorem still holds in this more general case. To do this, we will first be required to prove analogues of the classical asymptotic estimates of the Airy function. 


\section{The quantized Henon map and statement of Results}

Let $q$ be a real polynomial in one variable, and consider a version of the classical Henon map from $\mathbf{R}^{2}$ to $\mathbf{R}^{2}$ :

$$
\phi:(x, y) \mapsto\left(y+q^{\prime}(x),-x\right) .
$$

Our point of view will be to choose some quantization of this map and to study its dynamics, with a view to deciding whether the main features of the classical dynamics have analogues in the quantum version. It is well known (see $[\overline{B F}]$ ) that the Henon map cannot be embedded in the flow of a time-independent Hamiltonian on $\mathbf{R}^{2}$. Let, however,

$$
H(x, y, t)= \begin{cases}-q(x) & 0 \leq t<1 \\ \pi / 4\left(x^{2}+y^{2}\right) & 1 \leq t<2\end{cases}
$$

extended to $t \in \mathbf{R}$ by periodicity. Then it is easily shown that $\phi$ is the time- 2 map of the flow of $H$. Furthermore, $H$ has a natural quantization, which we also denote by $H \in \mathcal{A}\left(L^{2}(\mathbf{R})\right)$, given by

$$
H_{t}= \begin{cases}-q(x) & 0 \leq t<1 \\ \pi / 4\left(x^{2}-\frac{d^{2}}{d x^{2}}\right) & 1 \leq t<2\end{cases}
$$

Solving the Schrodinger equation

$$
\begin{aligned}
i \frac{\partial}{\partial t} V_{t} & =H_{t} \\
V_{0} & =\mathrm{Id}
\end{aligned}
$$

results in a unitary operator $V_{t}$ whose time- 2 map is

$$
V=V_{2}=e^{-i \pi / 4\left(x^{2}-\frac{d^{2}}{d x^{2}}\right)} e^{i q(x)}
$$

The first of these exponentials may be simplified by using the spectral form of the self-adjoint operator $x^{2}-\frac{d^{2}}{d x^{2}}$. This is the map

$$
\psi \mapsto \sum_{n=0}^{\infty}(2 n+1)\left(\psi, \phi_{n}\right),
$$

where the eigenfunctions $\phi_{n}$ are the elements of the standard Hermite basis of $L^{2}(\mathbf{R})$ :

$$
\begin{aligned}
\phi_{0}(x) & =e^{-x^{2} / 2}, \\
\phi_{n+1} & =(x-i y) \phi_{n},
\end{aligned}
$$

where we use $y$ here, and in what follows, to denote the self-adjoint operator $-i d / d x=F^{-1} x F$. Thus the spectral form of the unitary operator $e^{-i \pi / 4\left(x^{2}-\frac{d^{2}}{d x^{2}}\right)}$ is

$$
\begin{aligned}
\psi & \mapsto \sum_{n=0}^{\infty} e^{-i \pi / 4(2 n+1)}\left(\psi, \phi_{n}\right) \\
& =e^{-i \pi / 4} \sum_{n=0}^{\infty}(-i)^{n}\left(\psi, \phi_{n}\right),
\end{aligned}
$$


which is just the operator $e^{-i \pi / 4} F$. Ignoring the multiplicative constant $e^{-i \pi / 4}$, we take

$$
V=F e^{i q(x)}
$$

as our quantization of $\phi$.

A direct calculation shows that

$$
F=e^{i \pi / 4} e^{-i x^{2} / 2} e^{-i y^{2} / 2} e^{-i x^{2} / 2} .
$$

Thus

$$
e^{i x^{2} / 2} V e^{-i x^{2} / 2}=e^{i \pi / 4} e^{-i y^{2} / 2} e^{i\left(q(x)-x^{2}\right)} .
$$

In what follows, we will derive the spectral properties and dynamics of $V$ from those of

$$
U:=e^{-i y^{2} / 2} e^{i\left(q(x)-x^{2}\right)},
$$

which is its unitary conjugate up to the multiplicative constant.

Our aim is to prove the following theorems, which are proved in the remaining sections as Theorem 2.2 and Corollary 3.4, respectively:

Theorem 1.1. Let $q$ be a polynomial in one variable of odd degree at least three, with positive leading coefficient. Let $\mu: \mathbf{R} \rightarrow \mathbf{C}$ be defined by

$$
\mu(x)=\frac{1}{2 \pi} \int_{R} e^{i(q(t)-x t)} d t,
$$

interpreted in the principal value sense. Then $|\mu|$ is a continuous function that decays exponentially near $-\infty$ and rationally near $+\infty$.

Theorem 1.2. For $c>0$, let

$$
V_{c}=F e^{i(q(x)+c x)} \in \mathcal{U}\left(L^{2}(\mathbf{R})\right),
$$

where $q$ is a real polynomial in one variable of odd degree at least three, and with positive leading coefficient. Then for c sufficiently large, if $\psi \in L^{2}(\mathbf{R}), \epsilon>0$, and $r \in \mathbf{R}$, there exists an $N$ such that for all $n \geq N$, we have

$$
\begin{aligned}
\left\|V_{c}^{n} \psi\right\|_{(-\infty, r]} & <\epsilon, \\
\left\|F V_{c}^{n} \psi\right\|_{[-r, \infty)} & <\epsilon .
\end{aligned}
$$

In particular, $V_{c}^{n} \rightarrow 0$ as $n \rightarrow \infty$ in the weak operator topology, which implies that $V_{c}$ has purely continuous spectrum.

Note that $F e^{i(q(x)+c x)}$ quantizes the map $(x, y) \mapsto\left(y+q^{\prime}(x)+c,-x\right)$. For $c$ sufficiently large, all points are attracted to $(\infty,-\infty)$ by this map. Theorem 1.2 may be interpreted as a quantum analogue of this fact.

\section{Generalization of the Airy function, proof of Theorem 1.1}

The Airy function may be defined as the Fourier transform of the function $x \mapsto$ $e^{i x^{3}}$. This function is not in $L^{2}(\mathbf{R})$, of course, but the integral

$$
\frac{1}{\sqrt{2 \pi}} \int_{-\infty}^{\infty} e^{i\left(t^{3}-x t\right)} d t
$$

converges in the principal value sense for all $x \in \mathbf{R}$, so that the Airy function is the Fourier transform of $e^{i x^{3}}$ in say the space of tempered distributions. Beginning with this integral representation, one may represent the Airy function as a modified Bessel function of order $1 / 3$ Wa], and thereby deduce its asymptotic behavior on 
the real line. Fornaess and the present author made use of these properties of the Airy function in proving a special case of the present results [FW].

Applying the same techniques to the integral

$$
\mu(x):=F e^{i q}(x)=\frac{1}{\sqrt{2 \pi}} \int_{-\infty}^{\infty} e^{i(q(t)-x t)} d t
$$

leads to a complicated representation of the integral as a sum of hypergeometric functions, and delicate cancellations would be required to prove the desired decay properties by this route. Therefore we prefer to perform more direct asymptotic estimates of the integral. First we consider the behavior as $x \rightarrow-\infty$.

Lemma 2.1. Let

$$
q(x)=\sum_{j=0}^{d} a_{j} x^{j}
$$

be a polynomial of odd degree $d \geq 3$, and assume that $a_{d}>0$. Let $\mu$ be defined as above. Then there exists a constant $A>0$ such that

$$
|\mu(x)| \leq A e^{2 x} .
$$

Proof. It is easily verified that the contour of integration may be translated by $2 i$ without altering the integral, so that

$$
\begin{aligned}
\sqrt{2 \pi}|\mu(x)| & =\left|\int_{-\infty}^{\infty} e^{i(q(t+2 i)-x(t+2 i))} d t\right| \\
& \leq \int_{-\infty}^{\infty}\left|e^{i(q(t+2 i)-x(t+2 i))}\right| d t \\
& =\int_{-\infty}^{\infty} e^{\Re(i(q(t+2 i)-x(t+2 i)))} d t \\
& =e^{2 x} \int_{-\infty}^{\infty} e^{-2 d a_{d} t^{d-1}+O\left(t^{d-2}\right)} d t .
\end{aligned}
$$

Thus we may take

$$
A=\frac{1}{\sqrt{2 \pi}} \int_{-\infty}^{\infty} e^{-2 d a_{d} t^{d-1}+O\left(t^{d-2}\right)} d t
$$

which is finite because $a_{d}>0$ and $d$ is odd.

Next we consider the behavior of $\mu(x)$ as $x \rightarrow+\infty$. Assume that $x>0$, and assume that

$$
q(x)=\sum_{j=\eta}^{d} a_{j} x^{j}
$$

with $\eta \geq 2$; that is, that $a_{0}=a_{1}=0$. We lose nothing by this assumption, since $a_{0}$ has no effect on $|\mu|$ and changing $a_{1}$ just translates $\mu$. In the integral defining $\mu$, let us make the substitution $t \mapsto \zeta t$, where

$$
\zeta=\left(\frac{x}{d a_{d}}\right)^{\frac{1}{d-1}} .
$$


Here of course we are taking the positive real root. Then we have

$$
\begin{aligned}
\sqrt{2 \pi} \mu(x) & =\zeta \int_{-\infty}^{\infty} e^{i(q(\zeta t)-x(\zeta t))} d t \\
& =\zeta \int_{-\infty}^{\infty} e^{i\left(\zeta^{d} p_{\zeta}(t)\right)} d t,
\end{aligned}
$$

where

$$
p_{\zeta}(t)=\left(\sum_{j=\eta}^{d} a_{j} \zeta^{j-d} t^{j}\right)-d a_{d} t
$$

When $\zeta$ is large, the zeros of $p_{\zeta}^{\prime \prime}$ are clustered near $t=0$, while those of $p_{\zeta}^{\prime}$ are near $t= \pm 1$, so that the implicit function theorem gives us solutions $t_{1}=t_{1}(\zeta), \ldots, t_{n}=$ $t_{n}(\zeta)$ to $p_{\zeta}^{\prime}=0$, with $\left|t_{j}(\zeta)\right| \rightarrow 1$ as $\zeta \rightarrow \infty, j=1, \ldots, n$. Let $\beta_{0}=-\infty, \beta_{n}=\infty$, and $\beta_{j}=\left(t_{j}+t_{j+1}\right) / 2, j=1, \ldots, n-1$. Then

$$
\sqrt{2 \pi} \mu(x)=\zeta \sum_{j=1}^{n}\left(\int_{\beta_{j-1}}^{t_{j}} e^{i\left(\zeta^{d} p_{\zeta}(t)\right)} d t+\int_{t_{j}}^{\beta_{j}} e^{i\left(\zeta^{d} p_{\zeta}(t)\right)} d t\right) .
$$

We will show that the sum in this expression is $O\left(\zeta^{-d / 2}\right)$.

Making the change of variable $t \rightarrow-t$ if necessary, we see that each integral in the sum is of the form

$$
\int_{\alpha}^{\beta} e^{i\left(\zeta^{d} p_{\zeta}(t)\right)} d t
$$

or

$$
\int_{\alpha}^{\beta} e^{i\left(\zeta^{d} p_{\zeta}(-t)\right)} d t
$$

where $p_{\zeta}^{\prime}(\alpha)=0, p_{\zeta}^{\prime} \neq 0$ on $(\alpha, \beta], \alpha=\alpha(\zeta) \rightarrow 1$ as $\zeta \rightarrow \infty$, and either $\beta \equiv \infty$ or $\beta=\beta(\zeta) \rightarrow 1$ as $\zeta \rightarrow \infty$. We will consider the first type of integral. The reader will notice that in what follows we only require $a_{d} \neq 0$ rather than $a_{d}>0$; so the same proof will hold for the second type of integral.

Thus we are considering integrals of the form

$$
\int_{\alpha}^{\beta} e^{i\left(\zeta^{d} p_{\zeta}(t)\right)} d t
$$

where $p_{\zeta}^{\prime}(\alpha)=0, p_{\zeta}^{\prime} \neq 0$ on $(\alpha, \beta], \alpha=\alpha(\zeta) \rightarrow 1$ as $\zeta \rightarrow \infty$, and either $\beta \equiv \infty$ or $\beta=\beta(\zeta) \rightarrow 1$ as $\zeta \rightarrow \infty$. We will show that this integral is $O\left(\zeta^{-d / 2}\right)$ as $\zeta \rightarrow \infty$.

Assume without loss of generality that $p_{\zeta}^{\prime}>0$ in $I:=(\alpha, \beta]$. Then the map

$$
t \mapsto v=v_{\zeta}(t):=p_{\zeta}(t)-p_{\zeta}(\alpha)
$$

is injective in $I$, and making a change of variable we have

$$
\int_{\alpha}^{\beta} e^{i\left(\zeta^{d} p_{\zeta}(t)\right)} d t=e^{i \zeta\left(p_{\zeta}(\alpha)\right)} \int_{0}^{\gamma} e^{i \zeta^{d} v} f_{\zeta}(v) d v
$$

where

$$
\gamma=\gamma(\zeta)=p_{\zeta}(\beta(\zeta))-p_{\zeta}(\alpha(\zeta))
$$


(or $\gamma \equiv \infty$ if $\beta=\infty$ ) and

$$
\begin{aligned}
f_{\zeta}(v) & =\left(v_{\zeta}^{-1}\right)^{\prime}(v) \\
& =\frac{1}{p_{\zeta}^{\prime}\left(p_{\zeta}^{-1}\left(v+p_{\zeta}(\alpha)\right)\right.}
\end{aligned}
$$

Note that either $\gamma \equiv \infty$, or $\gamma(\zeta) \rightarrow 0$ as $\zeta \rightarrow \infty$. Let us consider the behavior of $f_{\zeta}(v)$ as $v \rightarrow 0^{+}$. For $t$ near $\alpha$, we have

$$
p_{\zeta}(t)=p_{\zeta}(\alpha)+k_{\zeta}(t-\alpha)^{2}+O_{\zeta}(t-\alpha)^{3},
$$

where

$$
\begin{aligned}
k_{\zeta} & =\frac{p_{\zeta}^{\prime \prime}(\alpha)}{2} \\
& =\frac{1}{2} \sum_{j=\eta}^{d} j(j-1) a_{j} \zeta^{j-d} \alpha^{j-2} \\
& =\frac{d(d-1) a_{d}}{2} \alpha^{d-2}+O_{\alpha}\left(\zeta^{-1}\right) .
\end{aligned}
$$

Note that, since $\alpha=\alpha(\zeta) \rightarrow \pm 1$ as $\zeta \rightarrow \infty$ and $a_{d} \neq 0$, it follows that $k_{\zeta} \rightarrow k_{\infty}$ as $\zeta \rightarrow \infty$, with $0<k_{\infty}<\infty$. In particular, we may assume that $k_{\zeta} \neq 0$. Now,

$$
\begin{aligned}
v_{\zeta}(t) & :=p_{\zeta}(t)-p_{\zeta}(\alpha) \\
& =k_{\zeta}(t-\alpha)^{2}+O_{\zeta}(t-\alpha)^{3} .
\end{aligned}
$$

Since the last term is bounded in $\zeta$, we may drop the subscript. And since

$$
\frac{v_{\zeta}(t)}{(t-\alpha)^{2}} \rightarrow k_{\zeta}
$$

as $t \rightarrow \alpha$, and $k_{\zeta} \neq 0$, it now follows that

$$
v_{\zeta}^{-1}(v)-\alpha=t-\alpha \sim\left(\frac{v}{k_{\zeta}}\right)^{1 / 2}
$$

as $v \rightarrow 0^{+}$. Since

$$
p_{\zeta}^{\prime}(t) \sim 2 k_{\zeta}(t-\alpha)
$$

as $t \rightarrow \alpha$, this gives

$$
\begin{aligned}
f_{\zeta}(v) & =\left(v_{\zeta}^{-1}\right)^{\prime}(v) \\
& =1 / v_{\zeta}^{\prime}\left(v_{\zeta}^{-1}(v)\right) \\
& =1 / p_{\zeta}^{\prime}(t) \\
& \sim 1 /\left[\left(\left(2 k_{\zeta}\right)\left(\frac{v}{k_{\zeta}}\right)^{1 / 2}\right)\right] \\
& =\left(\frac{1}{2\left(k_{\zeta}\right)^{1 / 2}}\right) v^{-1 / 2}
\end{aligned}
$$


as $v \rightarrow 0^{+}$. Furthermore, this relation may be differentiated. We have

$$
\begin{aligned}
f_{\zeta}^{\prime}(v) & =\frac{d}{d v}\left(\frac{1}{v_{\zeta}^{\prime}\left(v_{\zeta}^{-1}(v)\right)}\right) \\
& =-\frac{1}{\left(v_{\zeta}^{\prime}\left(v_{\zeta}^{-1}(v)\right)\right)^{2}} \cdot v_{\zeta}^{\prime \prime}\left(v_{\zeta}^{-1}(v)\right) \cdot\left(v_{\zeta}^{-1}\right)^{\prime}(v) \\
& =-\left(f_{\zeta}(v)\right)^{3} \cdot p_{\zeta}^{\prime \prime}\left(v_{\zeta}^{-1}(v)\right) \\
& \sim-\left[\frac{v^{\frac{1}{r}-1}}{2\left(k_{\zeta}\right)^{1 / 2}}\right]^{3} \cdot 2 k_{\zeta} \\
& =-\left(\frac{1}{4\left(k_{\zeta}\right)^{1 / 2}}\right) v^{-3 / 2}
\end{aligned}
$$

Note also that, for fixed $\zeta, f_{\zeta}^{\prime}(v)$ is integrable on any $[\kappa, \infty), \kappa>0$.

Now, write

$$
\int_{0}^{\gamma} e^{i \zeta^{d} v} f_{\zeta}(v) d v=\frac{1}{r k_{\zeta}^{1 / r}}(A-B)+C
$$

where

$$
\begin{aligned}
& A=\int_{0}^{\infty} e^{i \zeta^{d} v} v^{-1 / 2} d v, \\
& B=\int_{\gamma}^{\infty} e^{i \zeta^{d} v} v^{-1 / 2} d v,
\end{aligned}
$$

and

$$
C=\int_{0}^{\gamma} e^{i \zeta^{d} v} \phi_{\zeta}(v) d v
$$

and where

$$
\phi_{\zeta}(v)=f_{\zeta}(v)-\frac{v^{-1 / 2}}{2 k_{\zeta}^{1 / 2}}, \quad v \in(0, \gamma],
$$

recalling of course that we may have $\gamma=\infty$. Note that $\phi_{\zeta}(v)=o\left(v^{-1 / 2}\right)$, and $\phi_{\zeta}^{\prime}(v)=o\left(v^{-3 / 2}\right)$ as $v \rightarrow 0^{+}$. Furthermore, $\phi$ is continuous (this follows because $p_{\zeta}^{\prime} \neq 0$ on $\left.(\alpha, \beta]\right)$, and, in the case $\gamma=\infty, \phi_{\zeta}(v) \rightarrow 0$ as $v \rightarrow \infty$, and $\phi_{\zeta}^{\prime}(v)$ is integrable on any $[\kappa, \infty), \kappa>0$.

As a first step in estimating $A, B$, and $C$, let us investigate the behavior of $\gamma=\gamma(\zeta)$ as $\zeta \rightarrow \infty$. Recall that either $\gamma(\zeta) \equiv \infty$, or it is of the form

$$
\gamma(\zeta)=p_{\zeta}\left(\frac{1}{2}\left(t_{1}(\zeta)+t_{2}(\zeta)\right)\right)-p_{\zeta}\left(t_{1}(\zeta)\right)
$$

where $t_{1}(\zeta)<t_{2}(\zeta)$ are solutions of $p_{\zeta}^{\prime}=0$. Consider the case $\gamma(\zeta) \not \equiv \infty$. Since $t_{1}(\zeta), t_{2}(\zeta) \rightarrow \pm 1$ as $\zeta \rightarrow \infty$, we either have $\gamma(\zeta) \rightarrow 1$ or $\gamma(\zeta) \rightarrow 0$. Let us consider the latter case; that is, suppose without loss of generality that $t_{1}(\zeta), t_{2}(\zeta) \rightarrow 1$ as $\zeta \rightarrow \infty$. (This presupposes that $\eta<d$ in the expression

$$
p_{\zeta}(t)=\left(\sum_{j=\eta}^{d} a_{j} \zeta^{j-d} t^{j}\right)-d a_{d} t,
$$


for in that case the roots of $p_{\zeta}^{\prime}=0$ are exactly -1 and 1 , which implies that $\gamma=1$ or $\gamma=\infty$.) Note that

$$
p_{\zeta}^{\prime}(t)=d a_{d}\left(t^{d-1}-1\right)+O_{t}\left(\zeta^{\delta}\right)
$$

for some $\eta-d \leq \delta \leq-1$. Thus

$$
p_{\zeta}^{\prime}\left(t_{i}(\zeta)\right)-p_{\zeta}^{\prime}(1)=O\left(\zeta^{\delta}\right)
$$

The left-hand-side of this equation is equal to

$$
p_{\zeta}^{\prime \prime}(u(\zeta))\left(t_{i}(\zeta)-1\right)
$$

for some $u(\zeta)$ between 1 and $t_{i}(\zeta)$. Since $p_{\zeta}^{\prime \prime}(1)=d(d-1) a_{d}+O\left(\zeta^{\delta}\right)$ and $a_{d} \neq 0$, we have $p_{\zeta}^{\prime \prime}(u(\zeta))=O(1)$ as $\zeta \rightarrow \infty$. Thus

$$
\begin{aligned}
t_{i}(\zeta)-1 & =\frac{O\left(\zeta^{\delta}\right)}{O(1)} \\
& =O\left(\zeta^{\delta}\right),
\end{aligned}
$$

and it follows easily that

$$
\gamma(\zeta)=O\left(\zeta^{\delta}\right)
$$

Now, it is an easy exercise to show that

$$
\begin{aligned}
A & =e^{i \pi / 4} \Gamma(1 / 2) \zeta^{-d / 2} \\
& =O\left(\zeta^{-d / 2}\right) .
\end{aligned}
$$

Furthermore, if $\gamma \not \equiv-\infty$ (in which case $B=0$ ), a simple integration by parts shows that

$$
\begin{aligned}
|B| & =\left|\int_{\gamma}^{\infty} e^{i \zeta^{d} v} v^{-1 / 2} d v\right| \\
& =\left|\left[\frac{e^{i \zeta^{d} v}}{i \zeta^{d}} v^{-1 / 2}\right]_{\gamma}^{\infty}+\frac{1 / 2}{i \zeta^{d}} \int_{\gamma}^{\infty} e^{i \zeta^{d} v} v^{-1 / 2} d v\right| \\
& \leq \frac{\gamma^{-1 / 2}}{\zeta^{d}}+\frac{1}{2 \zeta^{d}} \int_{\gamma}^{\infty} v^{-3 / 2} d v \\
& =\frac{2 \gamma^{-1 / 2}}{\zeta^{d}} .
\end{aligned}
$$

In the case where $\gamma(\zeta) \rightarrow 1$ as $\zeta \rightarrow \infty$, the last line is $O\left(\zeta^{-d}\right)$. If $\gamma(\zeta) \rightarrow 0$, we get

$$
\begin{aligned}
|B| & <\frac{O\left(\zeta^{\delta(-1 / 2)}\right)}{\zeta^{d}} \\
& =O\left(\zeta^{\delta(-1 / 2)-d}\right) \\
& \leq O\left(\zeta^{(\eta-d)(-1 / 2)-d}\right) \\
& =O\left(\zeta^{(-d / 2)-(\eta / 2)}\right) \\
& <O\left(\zeta^{-d / 2}\right) .
\end{aligned}
$$

Thus $B=O\left(\zeta^{-d / 2}\right)$ in any case. It remains to consider $C$. The convergence of this improper integral at its lower limit follows from $\phi_{\zeta}(v)=o\left(v^{-1 / 2}\right)$ as $v \rightarrow 0^{+}$, and 
its convergence at its upper limit follows from an integration by parts and the fact that $\phi_{\zeta}^{\prime}$ is integrable near $\infty$. Choose $\kappa>0$ so small that

$$
\left|\phi_{\zeta}(v)\right|<v^{-1 / 2}, \quad\left|\phi^{\prime}(v)\right|<v^{-3 / 2}
$$

for $v \leq \kappa$. This choice may be made independent of $\zeta$.

Recall that if $\gamma(\zeta) \rightarrow 0$ as $\zeta \rightarrow \infty$, then $d>\eta$, and $\gamma(\zeta)=O\left(\zeta^{\delta}\right)$, where $\eta-d \leq \delta \leq-1$. In particular, $\gamma(\zeta)$ converges to zero more slowly than $\zeta^{-d}$. In all cases, therefore, we may assume that $\zeta^{-d} \in(0, \gamma(\zeta))$, and subdivide the integration range there. We may also assume that $\zeta^{-d}<\kappa$. Thus

$$
|C| \leq\left|\int_{0}^{\zeta^{-d}} e^{i \zeta^{d} v} \phi_{\zeta}(v) d v\right|+\left|\int_{\zeta^{-d}}^{\gamma(\zeta)} e^{i \zeta^{d} v} \phi_{\zeta}(v) d v\right| .
$$

For the first term on the right, we have

$$
\begin{aligned}
\left|\int_{0}^{\zeta^{-d}} e^{i \zeta^{d} v} \phi_{\zeta}(v) d v\right| & <\int_{0}^{\zeta^{-d}} v^{-1 / 2} d v \\
& =2 \zeta^{-d / 2} \\
& =O\left(\zeta^{-d / 2}\right) .
\end{aligned}
$$

For the second term, integration by parts gives

$$
\left|\int_{\zeta^{-d}}^{\gamma(\zeta)} e^{i \zeta^{d} v} \phi_{\zeta}(v) d v\right| \leq \zeta^{-d}\left[\left|\phi_{\zeta}(\gamma(\zeta))\right|+\left|\phi_{\zeta}\left(\zeta^{-d}\right)\right|+\left|\int_{\zeta^{-d}}^{\gamma(\zeta)} e^{i \zeta^{d} v} \phi_{\zeta}^{\prime}(v) d v\right|\right] .
$$

The first summand on the right is bounded in $\zeta$ in the cases $\gamma(\zeta) \rightarrow 1$ and $\gamma(\zeta) \equiv \infty$. Otherwise, we have

$$
\begin{aligned}
\left|\phi_{\zeta}(\gamma(\zeta))\right| & =o\left(\zeta^{-\delta / 2}\right) \\
& \leq o\left(\zeta^{(d-\eta) / 2}\right) \\
& <o\left(\zeta^{d / 2}\right) .
\end{aligned}
$$

The second summand is $o(d / 2)$. For the integral, in the case where $\gamma(\zeta) \rightarrow 0$, we may assume that $\gamma<\kappa$, obtaining

$$
\begin{aligned}
\left|\int_{\zeta^{-d}}^{\gamma(\zeta)} e^{i \zeta^{d} v} \phi_{\zeta}^{\prime}(v) d v\right| & <\int_{\zeta^{-d}}^{\gamma(\zeta)} v^{-3 / 2} d v \\
& <2 \zeta^{d / 2} \\
& =O\left(\zeta^{d / 2}\right) .
\end{aligned}
$$

In the other cases, subdivide the integration range at $\kappa$ to get

$$
\begin{aligned}
\left|\int_{\zeta^{-d}}^{\gamma(\zeta)} e^{i \zeta^{d} v} \phi_{\zeta}^{\prime}(v) d v\right| & =\left|\int_{\zeta^{-d}}^{\kappa} e^{i \zeta^{d} v} \phi_{\zeta}^{\prime}(v) d v\right|+\left|\int_{\kappa}^{\gamma(\zeta)} e^{i \zeta^{d} v} \phi_{\zeta}^{\prime}(v) d v\right| \\
& \leq \int_{\zeta^{-d}}^{\kappa} v^{-3 / 2} d v+\int_{\kappa}^{\gamma(\zeta)}\left|\phi_{\zeta}^{\prime}(v)\right| d v \\
& =O\left(\zeta^{d / 2}\right)+K \\
& =O\left(\zeta^{d / 2}\right),
\end{aligned}
$$


where

$$
K:=\int_{\kappa}^{\infty}\left|\phi_{\zeta}^{\prime}(v)\right| d v<\infty,
$$

because of the integrability of $\phi_{\zeta}^{\prime}$. Combining these terms and multiplying by $\zeta^{-d}$ gives

$$
\left|\int_{\zeta^{-d}}^{\gamma(\zeta)} e^{i \zeta^{d} v} \phi_{\zeta}(v) d v\right|=O\left(\zeta^{-d / 2}\right) .
$$

Thus $C=O\left(\zeta^{-d / 2}\right)$. Recall that we had

$$
\begin{aligned}
\int_{\alpha}^{\beta} e^{i\left(\zeta^{d} p_{\zeta}(t)\right)} d t & =e^{i \zeta\left(p_{\zeta}(\alpha)\right)} \int_{0}^{\gamma} e^{i \zeta^{d} v} f_{\zeta}(v) d v \\
& =e^{i \zeta\left(p_{\zeta}(\alpha)\right)} \frac{1}{r k_{\zeta}^{1 / r}}(A-B)+C
\end{aligned}
$$

Since $k_{\zeta} \rightarrow k_{\infty} \neq 0$ as $\zeta \rightarrow \infty$, we have shown that this integral is $O\left(\zeta^{-d / 2}\right)$. Since, in turn, $\mu(x)$ was $\zeta / \sqrt{2 \pi}$ times a finite sum of integrals of this form, we have

$$
\begin{aligned}
\mu(x) & =O\left(\zeta^{1-(d / 2)}\right) \\
& =O\left(x^{(1-(d / 2))(d-1)}\right) \\
& =O\left(x^{\frac{-(d-2)(d-1)}{2}}\right)
\end{aligned}
$$

as $x \rightarrow \infty$. We summarize this discussion, along with Lemma 2.1, in the following theorem:

Theorem 2.2. Let

$$
q(x)=\sum_{j=0}^{d} a_{j} x^{j}
$$

be a polynomial of odd degree $d$, and assume that $a_{d}>0$. Let

$$
\mu(x)=F e^{i q}(x)=\frac{1}{\sqrt{2 \pi}} \int_{-\infty}^{\infty} e^{i(q(t)-x t)} d t .
$$

Then $\mu$ is continuous, and there exist constants $C>0$ and $A>0$ such that

$$
|\mu(x)| \leq \max \left\{A e^{2 x}, C x^{\frac{-(d-2)(d-1)}{2}}\right\} .
$$

\section{Proof of Theorem 1.2}

In this section, when $E \subset \mathbf{R}$ is measurable and $\psi \in L^{2}(\mathbf{R})$, define

$$
\|\psi\|_{E}=\left(\int_{E}|\psi|^{2}\right)^{1 / 2} \text {. }
$$

Also, when $f: \mathbf{R} \rightarrow \mathbf{R}$ is continuous, we denote by $f(y)$ the convolution operator $F^{-1} f(x) F$.

Lemma 3.1. Let

$$
q(x)=\sum_{j=0}^{d} a_{j} x^{j}
$$

be a polynomial of odd degree $d$, and assume that $a_{d}>0$. Let $U_{c}$ be the operator

$$
U_{c}:=e^{-i y^{2} / 2} e^{i(q(x)+c x)}=F^{-1} e^{-i x^{2} / 2} F e^{i(q(x)+c x)}: L^{2}(\mathbf{R}) \rightarrow L^{2}(\mathbf{R}),
$$


and for $k \in \mathbf{R}$ define

$$
\mathcal{C}_{k}=\left\{\psi \in L^{2}(\mathbf{R}):|\psi(x)|<e^{x-k} \text { for } x<k\right\} .
$$

Then for $c>0$ sufficiently large, there exists $\delta>0$ such that

$$
U_{c}: F^{-1} \mathcal{C}_{k} \rightarrow F^{-1} \mathcal{C}_{k+\delta} .
$$

Proof. Let $\mu=F\left(e^{i(q(x)+c x)}\right)$. By Theorem 2.1 and elementary properties of the Fourier transform, $F\left(e^{i q}\right)$ is bounded on $\mathbf{R}$ by $\max \left\{T, A e^{2 x}\right\}$ for some $T>0$, $A>0$. Then by elementary properties of the Fourier transform, $\mu$ is bounded by $\max \left\{T, A e^{2(x-c)}\right\}$. Assume that $c$ is sufficiently large that

$$
\delta:=c-(1 / 2) \log A-\log (T+1)>0 .
$$

Suppose that $\psi \in F^{-1} \mathcal{C}_{k}, k \in \mathbf{R}$. Then for $x<k+\delta$, we have

$$
\begin{aligned}
\left|F U_{c} \psi(x)\right| & =\left|F e^{-i y^{2} / 2} e^{i(q(x)+c x)} \psi(x)\right| \\
& =\left|e^{-i x^{2} / 2} F e^{i(q(x)+c x)} \psi(x)\right| \\
& =|(\mu *(F \psi))(x)| \\
& =\left|\int_{\mathbf{R}} \mu(x-t) F \psi(t) d t\right| \\
& \leq \int_{\mathbf{R}}|\mu(x-t) F \psi(t)| d t \\
& \leq D_{1}+D_{2}+D_{3},
\end{aligned}
$$

where

$$
\begin{aligned}
& D_{1}=\left|\int_{-\infty}^{x-c+(1 / 2) \log A} \mu(x-t) F \psi(t) d t\right| \\
& D_{2}=\left|\int_{x-c+(1 / 2) \log A}^{k} \mu(x-t) F \psi(t) d t\right| \\
& D_{3}=\left|\int_{k}^{\infty} \mu(x-t) F \psi(t) d t\right| .
\end{aligned}
$$

Then

$$
\begin{gathered}
D_{1} \leq \int_{-\infty}^{x-c+(1 / 2) \log A} T e^{t-k} d t \\
=e^{x-k-c+(1 / 2) \log A+\log T}, \\
D_{2} \leq \int_{x-c+(1 / 2) \log A}^{k} e^{t-k} \cdot A e^{2(x-c-t)} d t \\
=e^{x-c-k+(1 / 2) \log A}-A e^{2(x-k-c)},
\end{gathered}
$$


and

$$
\begin{aligned}
D_{3} & \leq\left(\int_{k}^{\infty}|F \psi(t)|^{2} d t\right)^{1 / 2}\left(\int_{k}^{\infty}|\mu(x-t)|^{2} d t\right)^{1 / 2} \\
& \leq 1 \cdot\left(\int_{k}^{\infty}|\mu(x-t)|^{2} d t\right)^{1 / 2} \\
& \leq\left(\int_{k}^{\infty} A^{2} e^{4(x-c-t)} d t\right)^{1 / 2} \\
& =\frac{A}{2} e^{2(x-k-c)} \\
& <A e^{2(x-k-c)},
\end{aligned}
$$

so that

$$
\begin{aligned}
D_{1}+D_{2}+D_{3} & \leq e^{x-k-c+(1 / 2) \log A+\log T}+e^{x-c-k+(1 / 2) \log A} \\
& =e^{x-(k+\delta)}\left(e^{\log T-\log (T+1)}+e^{-\log (T+1)}\right) \\
& =e^{x-(k+\delta)} .
\end{aligned}
$$

Now, let $q_{1}(x)=q(x)+x^{2} / 2$. Again applying Theorem 2.1, we obtain real numbers $T_{1}, A_{1}$ such that, if $\mu_{1}=F q_{1}$, then $\left|\mu_{1}(x)\right|<\max \left\{T_{1}, A_{1} e^{2 x}\right\}$ for all $x \in \mathbf{R}$.

Lemma 3.2. Under the same hypotheses as in the previous lemma, there exists a $c>0$ such that the following holds: if $\psi \in F^{-1} \mathcal{C}_{k}$ for some $k \in R$, then for any $r \in \mathbf{R}$, there exists an $N$ such that $U_{c}^{n} \psi \in \mathcal{C}_{r}$ for all $n \geq N$.

Proof. Choosing the $c$ provided by the previous lemma, we may assume by applying $U_{c}$ a finite number of times that $\psi \in F^{-1} \mathcal{C}_{r^{\prime}}$, where

$$
r^{\prime}:=\max \left\{r, r+\log \left(T_{1}+A_{1} e^{-2 c}\right)\right\}
$$

Then

$$
\begin{aligned}
\left|U_{c} \psi(x)\right| & =\left|e^{-i y^{2} / 2} e^{i(q(x)+c x)} \psi(x)\right| \\
& =\left|e^{i x^{2} / 2}\left(e^{-i x^{2} / 2} e^{-i y^{2} / 2} e^{-i x^{2} / 2}\right) e^{i\left(q_{1}(x)+c x\right)} \psi(x)\right| \\
& =\left|F e^{i\left(q_{1}(x)+c x\right)} \psi(x)\right| \\
& =\left|\left(\mu_{1} *(F \psi)\right)(x)\right| .
\end{aligned}
$$

Suppose now that $x<r^{\prime}$. Then

$$
\left|U_{c} \psi(x)\right| \leq D_{1}+D_{2}+D_{3}
$$


where

$$
\begin{aligned}
& D_{1}=\left|\int_{-\infty}^{x} \mu_{1}(x-t) F \psi(t) d t\right| \\
& D_{2}=\left|\int_{x}^{r^{\prime}} \mu_{1}(x-t) F \psi(t) d t\right| \\
& D_{3}=\left|\int_{r^{\prime}}^{\infty} \mu_{1}(x-t) F \psi(t) d t\right| .
\end{aligned}
$$

Then

$$
\begin{gathered}
D_{1} \leq \int_{-\infty}^{x} T_{1} e^{t-r^{\prime}} d t \\
=T_{1} e^{x-r^{\prime}}, \\
D_{2} \leq \int_{x}^{r^{\prime}} e^{t-r^{\prime}} \cdot A_{1} e^{2(x-c-t)} d t \\
=A_{1}\left(e^{x-r^{\prime}-2 c}-e^{2\left(x-r^{\prime}-c\right)}\right),
\end{gathered}
$$

and

$$
\begin{aligned}
D_{3} & \leq\left(\int_{r^{\prime}}^{\infty}|F \psi(t)|^{2} d t\right)^{1 / 2}\left(\int_{r^{\prime}}^{\infty}\left|\mu_{1}(x-t)\right|^{2} d t\right)^{1 / 2} \\
& \leq 1 \cdot\left(\int_{r^{\prime}}^{\infty}\left|\mu_{1}(x-t)\right|^{2} d t\right)^{1 / 2} \\
& \leq\left(\int_{r^{\prime}}^{\infty} A_{1}^{2} e^{4(x-c-t)} d t\right)^{1 / 2} \\
& =\frac{A_{1}}{2} e^{2\left(x-r^{\prime}-c\right)} \\
& <A_{1} e^{2\left(x-r^{\prime}-c\right)}
\end{aligned}
$$

so that

$$
\begin{aligned}
D_{1}+D_{2}+D_{3} & \leq e^{x-r^{\prime}}\left(T_{1}+A_{1} e^{-2 c}\right) \\
& =e^{x-\left(r^{\prime}-\log \left(T_{1}+A_{1} e^{-2 c}\right)\right)} \\
& \leq e^{x-r} .
\end{aligned}
$$

Theorem 3.3. For real $c$, consider the operator $V_{c}:=F e^{i q(x)+c x}$, where $q$ is a polynomial of odd degree at least three, and with positive real leading coefficient. Then for c sufficiently large, for any $\psi \in L^{2}(\mathbf{R}), \epsilon>0$, and $r \in \mathbf{R}$ there exists an $N$ such that

$$
\operatorname{dist}\left(V_{c}^{n} \psi, \mathcal{C}_{r}\right)<\epsilon, \quad \operatorname{dist}\left(V_{c}^{n} \psi, F \mathcal{C}_{r}\right)<\epsilon
$$

for all $n \geq N$, where

$$
\mathcal{C}_{k}=\left\{\psi \in L^{2}(\mathbf{R}):|\psi(x)|<e^{x-k} \text { for } x<k\right\} .
$$


Proof. Consider

$$
\begin{aligned}
U_{c} & :=e^{i x^{2} / 2} V_{c} e^{-i x^{2} / 2} \\
& =e^{i x^{2} / 2}\left(e^{i \pi / 4} e^{-i x^{2} / 2} e^{-i y^{2} / 2} e^{-i x^{2} / 2}\right) e^{i\left(q(x)-x^{2} / 2+c x\right)} \\
& =e^{i \pi / 4} e^{-i y^{2} / 2} e^{i\left(q(x)-x^{2}+c x\right)} .
\end{aligned}
$$

Now,

$$
X:=\bigcup_{k \in \mathbf{R}} \mathcal{C}_{k}
$$

is a dense subset of $L^{2}(\mathbf{R})$, and so therefore is any unitary image of $X$. It suffices therefore to prove the theorem under the assumption that $\psi \in e^{-i x^{2} / 2} F^{-1} X$; that is, we assume that there exists $k \in \mathbf{R}$ such that

$$
e^{i x^{2} / 2} \psi \in F^{-1} \mathcal{C}_{k} .
$$

Now, let $r \in R$. We may apply Lemma 3.2 to obtain a $c$ and an $N$ such that

$$
U_{c}^{n} e^{i x^{2} / 2} \psi \in \mathcal{C}_{r}
$$

for all $n \geq N-1$. But then

$$
\begin{aligned}
\left|V_{c}^{n} \psi(x)\right| & =\left|e^{-i x^{2} / 2} U_{c}^{n} e^{i x^{2} / 2} \psi(x)\right| \\
& =\left|U_{c}^{n} e^{i x^{2} / 2} \psi(x)\right|,
\end{aligned}
$$

so that $V_{c}^{n} \psi \in \mathcal{C}_{r}$. Then

$$
\begin{aligned}
\left|F V_{c}^{n+1} \psi(x)\right| & =\left|F^{2} e^{i(q(x)+c x)} V_{c}^{n} \psi(x)\right| \\
& =\left|e^{i(q(-x)-c x)} F^{2} V_{c}^{n} \psi(x)\right| \\
& =\left|F^{2} V_{c}^{n} \psi(x)\right|,
\end{aligned}
$$

so that $V_{c}^{n+1} \psi \in F \mathcal{C}_{r}$.

Corollary 3.4. Using the real number c provided by the theorem, if $\psi \in L^{2}(\mathbf{R})$, $\epsilon>0$, and $r \in \mathbf{R}$, there exists an $N$ such that for all $n \geq N$, we have

$$
\begin{aligned}
\left\|V_{c}^{n} \psi\right\|_{(-\infty, r]} & <\epsilon \\
\left\|F V_{c}^{n} \psi\right\|_{[-r, \infty)} & <\epsilon .
\end{aligned}
$$

In particular, $V_{c}^{n} \rightarrow 0$ as $n \rightarrow \infty$ in the weak operator topology, which implies that $V_{c}$ has purely continuous spectrum.

Proof. Let $\rho=r-\log (\epsilon / \sqrt{2})$. Applying the theorem, we find an $N$ such that for all $n \geq N$,

$$
\operatorname{dist}\left(V_{c}^{n} \psi, \mathcal{C}_{r}\right)<\frac{\epsilon}{2}, \operatorname{dist}\left(V_{c}^{n} \psi, F \mathcal{C}_{r}\right)<\frac{\epsilon}{2} .
$$

Furthermore, for all $\phi \in \mathcal{C}_{\rho}$, we have

$$
\begin{aligned}
\|\phi\|_{(-\infty, r]} & <\left(\int_{-\infty}^{r} e^{2(x-\rho)} d x\right)^{1 / 2} \\
& =(1 / \sqrt{2}) e^{r-\rho} \\
& =\frac{\epsilon}{2}
\end{aligned}
$$


and for all $\phi \in F \mathcal{C}_{\rho}$, we have

$$
\begin{aligned}
\|F \phi\|_{[-r, \infty)} & =\left\|F^{2} F^{-1} \phi\right\|_{[-r, \infty)} \\
& =\left\|F^{-1} \phi\right\|_{(-\infty, r]} \\
& <\left(\int_{-\infty}^{r} e^{2(x-\rho)} d x\right)^{1 / 2} \\
& =\frac{\epsilon}{2} .
\end{aligned}
$$

The corollary now follows.

\section{REFERENCES}

[AS] J. Avron and B. Simon. Transient and recurrent spectrum. J. Funct. Anal. 43, (1981). MR 83c: 47008

[Be] J. Bellissard. Stability and instability in quantum mechanics. Trends and Developments in the Eighties, World Sci. Publishing, Singapore, 1985. MR 87i:81046

[BF] G. Buzzard and J.E. Fornaess. Compositional roots of Henon maps. Geometric complex analysis (Hayama, 1995), World Sci. Publishing, River Edge, NJ, 1996. MR 98d:32032

[CG] L. Carleson and T. W. Gamelin. Complex Dynamics. Springer-Verlag, 1993. MR 94h:30033

[Ll] R. de la Llave. A tutorial on KAM theory. Proc Sympos. Pure Math. 69 (2001). MR 2002h:37123

[Fo] J. E. Fornæss. Infinite-dimensional complex dynamics: quasiconjugacies, localization, and quantum chaos. Disc. Cont. Dyn. Syst. 6, 1 (2000). MR 2000k:37062

[FW] J. E. Fornæss and B. Weickert. A quantized Henon map. Disc. Cont. Dyn. Syst. 6, 3 (2000). MR 2001k:37135

[KS] A. Knauf and Y. G. Sinai. Classical nonintegrability, quantum Chaos. Birkhauser, 1997. MR 98k:58181

[RS] F. Riesz and B. Sz-Nagy. Functional Analysis. Frederick Ungar Publishing, 1955. MR 17:175i

[Wa] G.N. Watson. A treatise on the Theory of Bessel Functions, 2nd ed. Cambridge University Press, 1966.

[W1] B. Weickert. Infinite-dimensional complex dynamics: a quantum random walk. Disc. Cont. Dyn. Syst. 7, 3 (2001). MR 2002a:82078

[W2] B. Weickert. Quantizations of linear self-maps of $\mathbf{R}^{2}$. Acta. Sci. Math., to appear.

Department of Mathematics, Washington and Lee University, Lexington, Virginia 24450

E-mail address: weickertb@wlu.edu 